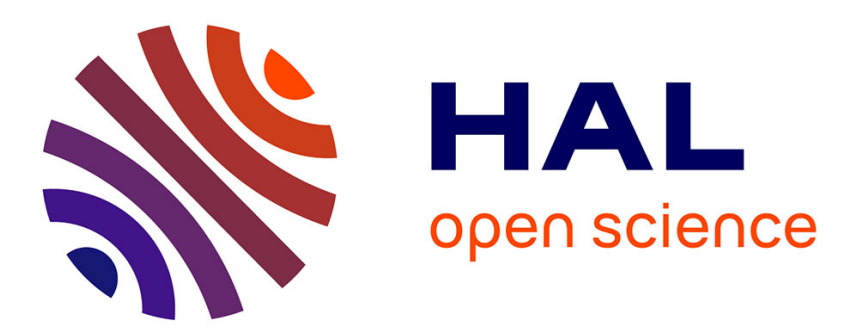

\title{
Éduquer à l'égalité des sexes à l'école primaire: autour de quelques gestes professionnels accompagnant une activité de lecture littéraire
}

Gaël Pasquier, Cendrine Marro, Laurence Breton

\section{To cite this version:}

Gaël Pasquier, Cendrine Marro, Laurence Breton. Éduquer à l'égalité des sexes à l'école primaire: autour de quelques gestes professionnels accompagnant une activité de lecture littéraire. Le Français Aujourd'hui, 2016. hal-02510470

\author{
HAL Id: hal-02510470 \\ https://hal.science/hal-02510470
}

Submitted on 17 Mar 2020

HAL is a multi-disciplinary open access archive for the deposit and dissemination of scientific research documents, whether they are published or not. The documents may come from teaching and research institutions in France or abroad, or from public or private research centers.
L'archive ouverte pluridisciplinaire HAL, est destinée au dépôt et à la diffusion de documents scientifiques de niveau recherche, publiés ou non, émanant des établissements d'enseignement et de recherche français ou étrangers, des laboratoires publics ou privés. 


\title{
ÉDUQUER À L'ÉGALITÉ DES SEXES À L'ÉCOLE PRIMAIRE :
}

\section{AUTOUR DE QUELQUES GESTES PROFESSIONNELS ACCOMPAGNANT UNE ACTIVITÉ DE LECTURE LITTÉRAIRE}

\author{
Gaël PASQUIER \\ Université Paris Est Créteil \\ Laboratoire LIRTES, OUIEP
}

\section{Cendrine MARR0}

Université Paris Ouest Nanterre La Défense

Laboratoire LEGS

\section{Laurence BRETON}

Direction académique des Hauts-de-Seine

Mission « Maitrise de la langue et enseignement moral et civique »

Que peut signifier concrètement, en termes de gestes professionnels, sensibiliser et former des professeur-e-s des écoles à l'égalité des sexes, de manière à ce qu'ils/elles puissent mettre en œuvre, dans leur classe, cette éducation'? C'est en vue de trouver des éléments de réponses concrets à cette question que nous avons mis en place une recherche-action en septembre 2013 impliquant chaque année une douzaine de professeur-e-s des écoles ${ }^{2}$.

Sachant, de par nos expériences de formateur-trice d'enseignant-e-s et de chercheurs et chercheuses spécialisé-e-s sur cette thématique, d'une part, qu'une des résistances à cette éducation est sa perception comme tâche supplémentaire qui vient alourdir un programme déjà bien chargé (Pasquier 2013) et, d'autre part, que les œuvres littéraires étudiées en classe sont souvent loin d'être dénuées de sexisme (Brugeilles et al. 2009 ; Dafflon Novelle 2006), nous avons choisi comme support d'activité la littérature

\footnotetext{
1. Le site Eduscol rappelle en ces termes cette obligation légale : "L'École compte parmi ses missions fondamentales celle de garantir l'égalité des chances des filles et des garçons ». C'est le sens des articles L. 121-1 et L. 312-17-1 du code de l'éducation qui disposent que l'École contribue, à tous les niveaux, à favoriser la mixité et l'égalité entre les femmes et les hommes, notamment en matière d'orientation, ainsi qu'à la prévention des préjugés sexistes et des violences faites aux femmes.

2. Ne sont officiellement inscrites dans le dispositif complet (recherche et formation) que des enseignantes ; toutefois un enseignant collabore à la recherche par le biais d'une de ses collègues en mettant en œuvre, dans sa classe, le dispositif élaboré dans les sessions de formation.
} 
jeunesse ${ }^{3}$. Ce choix nous a permis de travailler à la mise en ouvre d'une éducation à l'égalité des sexes pensée de manière intégrée à une activité régulière de classe : l'étude de textes littéraires. Pour ce faire, nous avons fixé comme objectif aux échanges avec les enseignant-e-s, l'organisation par ces dernier-ère-s de débats interprétatifs d'albums associés à l'élaboration d'un carnet de littérature par leurs élèves. Ce carnet vise à permettre aux élèves un retour réflexif personnel sur la lecture, éclairé par le débat, en lien avec l'égalité des sexes (Breton, Marro et Pasquier 2016).

Le présent article entend donner un aperçu du travail réalisé à partir de l'analyse de deux séances de débat menées dans une classe de CM2 (cinquième primaire) et une classe de $\mathrm{CP}$ (première primaire) d'une même école, analyse via laquelle seront interrogés certains gestes professionnels des enseignant-e-s, et esquissées des pistes de questionnements en lien avec le dispositif mis en place. Avant de développer ces deux points, nous préciserons, dans une première partie, notre conception de ce que signifie pour nous éduquer à l'égalité des sexes à l'école. Nous expliciterons en particulier en quoi le concept de genre nous est utile pour penser cette éducation et quel usage nous en faisons.

\section{Du concept de genre pour penser l'égalité des sexes à l'école : position conceptuelle et usage pratique}

Le cadre des études de genre nous permet non seulement de penser conceptuellement l'égalité des sexes mais aussi d'agir concrètement pour cette égalité dans le quotidien de la classe. Et ce, en particulier, au regard d'un certain nombre d'idéologies, au premier rang desquelles celle de LA différence des sexes, qui s'articule avec celle de leur nécessaire complémentarité qui légitime la contrainte à l'hétérosexualité (Rich 1980). Ces trois idéologies, extrêmement prégnantes, et leurs interrelations, étudiées et déconstruites par les études de genre, sont, pour nous, le ciment des inégalités dont elles favorisent l'acceptation. En effet, comme c'est le cas pour tout grand principe, la réalisation effective de l'égalité ne va pas de soi (Ferrand 2004). Il est donc important que les élèves puissent apprendre qu'il existe des lois censées la garantir, mais aussi de les accompagner à repérer ce qui persiste à lui faire obstacle dans la réalité quotidienne et les représentations communes (qui sont parfois aussi les leurs). C'est ici que se manifeste l'intérêt de recourir

3. Soulignons après d'autres que, d'une manière générale, si les activités de classe autour de la littérature jeunesse ne donnent pas toujours lieu à un réel travail sur le texte littéraire en lui-même (qualité du texte, rapport texte-illustration, univers de référence, implicite ou inférences, états mentaux des personnages ... (Inspection générale de l'Éducation nationale 2011 : 131) ; contrairement à ce que l'on pourrait penser tant ce fait est flagrant ; il ne donne pas davantage lieu, de manière un peu systématique, à une réflexion sur ce que véhicule ce texte, en termes de valeurs et de représentations, notamment sexuées. Or, ces dernières sont la plupart du temps peu diversifiées, sur un mode stéréotypé et surtout inégalitaire (Brugeilles et al. 2009 ; Dafflon Novelle 2006). 
au concept de genre comme concept opératoire pour penser l'éducation à l'égalité des sexes. Nous le mobilisons dans une acception sociopolitique, soit comme "système hiérarchisant de normes de sexes qui légitime les inégalités en les naturalisant sous les habits de LA différence "; sachant que ce dernier syntagme renvoie ici à tout ce que madame et monsieur Tout-le-monde pensent concernant ce en quoi filles et garçons, femmes et hommes, different "naturellement ", de manière dichotomique, tant sur un plan biologique que psychologique ou même social (Marro 2011, 2012).

Dans le cadre de notre recherche-action, l'explicitation de cette définition aux enseignant-e-s permet d'emblée d'introduire une manière différente de penser les deux sexes non pas en tant que catégories naturelles mais en tant que catégories sociales qui, à ce titre, sont porteuses de valeurs, de statuts et de pouvoirs socialement différenciés et ce faisant d'inégalités. Nous incitons ainsi les professeur-e-s avec lesquel-le-s nous travaillons à questionner la littérature de jeunesse en ne se contentant pas d'un inventaire des stéréotypes et préjugés sexistes qui l'habitent mais en se situant de manière privilégiée dans une " approche genre ", une approche critique qui travaille à repérer et à remettre en cause le déterminisme biologique fréquemment convoqué dans nombre d'albums pour parler des filles et des garçons (caractère, gout, capacités, etc.). Dans cette perspective nous les invitons à se centrer sur la dialectique différences-inégalités de sexe afin d'être à même de traduire en termes d'inégalités les différences mises en jeu. L'identification des enjeux de domination, de pouvoir, de reconnaissance sous-jacents aux différences de sexe évoquées, et autour desquels les individus sont plus ou moins explicitement en confrontation, permet de rendre visibles les rapports asymétriques de sexe dénommés rapports sociaux de sexe (Kergoat 2005) transmis par cette littérature.

Ces orientations conceptuelles ont des conséquences, tant au niveau du choix des albums $s^{4}$ que sur la manière dont vont être pensés et menés les débats. C'est précisément aux questionnements mis en ouvre dans ce cadre que nous allons nous intéresser ici. L'objectif est d'illustrer au sein de situations de classes concrètes comment opérationnaliser cette approche en repérant ce qui fait obstacle ou écran à sa mise en œuvre.

\section{Autour de deux débats réalisés en classe}

\section{En Cours moyen 2}

La première séance, réalisée en CM2 porte sur le l'album Marre du rose de N. Hense, illustré par I. Green (2009). Elle s'est déroulée en janvier et a été précédée de deux séances de débats et d'écriture à partir de deux saynètes extraites des Gouters philo, Les Garçons et les filles, de B. Labbé et C. Puech

4. Ce choix tend dans le cadre de ce dispositif à privilégier, mais non exclusivement, des albums qu'il est possible de définir comme "antisexistes ", c'est-à-dire qui contestent l'association du sexe d'état civil à des normes ou un rôle à jouer dans la société. 
(2001) : "Allez, pleure pas comme une fille ! " et " Des rôles qui empêchent ». Précisons que ces élèves, dans le cadre d'un projet de «médiation par les pair-e-s ", avait antérieurement travaillé sur l'identification et l'expression de leurs émotions ${ }^{5}$.

Marre du rose met en scène une héroïne qui dit aimer le noir à la différence des autres filles dont elle ne partage pas par ailleurs le gout pour les poupées, les princesses... Elle est confrontée aux remarques de ses parents qui la qualifient de "garçon manqué " et lui rappellent volontiers que les activités qui l'attirent ne sont pas pour elle. La difficulté que présente cet ouvrage, pour les professeur-e-s des écoles, comme pour les enfants, est de saisir que le refus de l'héroïne de porter du rose va bien au-delà du simple rejet d'une couleur. C'est le mécanisme même d'assignation sociale systématique de gouts, de préoccupations et de comportements spécifiques à un sexe qu'elle conteste. La question n'est donc pas tant de savoir si filles et garçons peuvent exercer des activités ou apprécier des choses habituellement attribuées à l'autre sexe, mais plus centralement de s'intéresser à ce que produit ce système d'attributions notamment en termes de contraintes psychologiques et ses conséquences à différents niveaux (ressentis personnels, relations inter individuelles...) sur celles et ceux qui le transgressent.

Durant toute cette séance, le questionnement de l'enseignant, en lien avec les pistes pédagogiques évoquées lors des temps de formation, s'est essentiellement centré sur le ressenti des élèves et l'empathie vis-à-vis des personnages principaux :

Qu'est-ce que vous avez ressenti ? [...] Quelles sont les émotions que vous avez perçues en écoutant cette histoire ? [...] Vous avez quelque chose à dire sur cet album qui vous a marqué ? / D'après vous, comment elle se sent cette jeune fille ? [...] Quels sentiments elle éprouve tout au long de l'histoire? [...] Quels sentiment d'après-vous ressent la jeune fille ou une personne lorsqu'on lui parle comme ça ? Quand on lui dit justement qu'elle est un garçon manqué ? [...] Pourquoi [les parents] disaient ça ? [...] Qu'est-ce que peuvent ressentir ces garçons [les deux camarades de l'héroïne] ? [...] Pourquoi elle se sent exaspérée? [...]

Si nous souhaitons nous intéresser plus précisément à ce débat, c'est que l'entrée choisie par l'enseignant nous semble permettre aux élèves d'élaborer une réflexion sur le système de genre (sans pour autant que ce terme ait été utilisé en classe) et ses conséquences en termes de production d'inégalités, d'injustices. Ses questions et relances conduisent en effet petit à petit à l'émergence, dans la discussion, de points de tension ou dilemmes en lien direct avec le texte et résonant également au-delà.

Un de ces premiers points de tension porte sur les différentes significations du mot normal. Le terme réapparait trois fois dans le débat dans la bouche de la même élève, Samia :

5. La séance dont il est question ici est animée par le titulaire de la classe et observée par un chercheur (séance enregistrée). 
[L'héroïne] est déçue parce qu'elle a envie d'être une fille normale ${ }^{6}$, comme les autres ; enfin, elle a pas envie qu'on la juge »; " moi, je connaissais une fille, au début elle était tranquille, c'était une fille normale; après, elle a commencé à jouer avec les garçons, et petit à petit, elle s'amusait avec eux tranquille "; "c'est comme si elle a envie d'être une fille normale, pas qu'on la juge, et qu'après les autres ils disent à des personnes qu'elle s'habille qu'en noir ».

Ces trois interventions posent dans le débat - sans pour autant que ce point ait été explicitement souligné - la question de savoir ce que veut dire normal. Est-ce que l'on est «normal » lorsque l'on reste conforme aux normes de sexe traditionnelles ou est-ce qu'une " fille normale " n'est pas tout simplement une personne qui agit selon ses gouts sans se préoccuper $\mathrm{du}$ qu'en-dira-t-on (par exemple en restant "tranquille») ? En d'autres termes, est-ce que le regard porté sur l'héroïne et les remarques auxquelles elle fait face (quand on lui dit qu'elle n'est pas " une vraie fille », sans pour autant la considérer comme un garçon, ce qu'elle ne cherche d'ailleurs pas à être), ne l'empêchent pas justement d'être une "fille normale ", c'est-à-dire selon ses aspirations personnelles, soit indépendamment des normes de sexe?

Ce questionnement est prolongé par certaines remarques faites par Younes au cours du débat. Celles-ci permettent de relativiser les normes de sexe selon les contextes, en reprenant des éléments du livre (aimer la couture quand on est un garçon, les grues quand on est une fille) mais en les éclairant à partir d'éléments nouveaux tirés de sa propre expérience :

Déjà quand on fait de la couture, c'est pas que pour les filles, puisque dans d'autres pays, y'a des personnes, y'a des garçons qui font de la couture. Et aussi quand son père il dit que la grue c'est pour les garçons, c'est pas que pour les garçons, puisque c'est un métier où y'a pas que des garçons, y’a aussi des filles.

Dès lors, à partir du ressenti de l'héroïne que les questions de l'enseignant-e-s mettent en avant, c'est un renversement du regard qui est introduit dans les échanges : la norme étant posée comme relative par les élèves, ce qui pose problème dans cette histoire ne sont plus tant les gouts de l'héroïne que le regard qui est porté sur eux, et la manière dont ils sont construits comme une déviance ; et ce d'autant plus que les individus ne sont pas par essence conformes ou non aux normes de sexes, mais jouent aussi avec les codes :

Par exemple, je sais plus comment il s'appelle celui-là [le personnage] qui fait de la couture avec ses poupées; il faisait de la couture mais en même temps il jouait à des trucs de garçon. Donc ça ne veut rien dire. Par exemple, lui on croyait que c'était une fille manquée, euh un garçon manqué, euh une

6. Nous soulignons. 
fille manquée ${ }^{7}$, mais c'est pas une fille manquée parce qu'en même temps il fait de la couture mais aussi il fait des trucs de garçon. Enfin il creuse avec la carte ${ }^{8}$. (Younes)

Un deuxième " point de tension " porte sur les mécanismes d'assujettissement et d'émancipation du sujet, notamment par le retournement du stigmate (Goffman 1975) dont on signifie à l'individu qu'il est porteur. À partir de l'expression "garçon manqué », le débat met en évidence les effets produits par les jugements portés sur autrui :

Elle doit être mal à l'aise parce que tout le monde dit : "ah t'es un garçon, t'es un garçon ", alors que juste, elle aime pas le rose. (Lucie)

Elle se sent triste. (Jennifer)

Vexée. (Sébastien)

Exaspérée. (Fatou)...

Certains élèves mettent également en avant que l'expression "garçon manqué " n'est pas un simple constat. Au moment même où elle est formulée, elle entend signifier à la personne à qui elle s'adresse " ce qu'elle est vraiment ». Elle la définit donc en même temps qu'elle la désigne à l'ensemble des personnes présentes (Eribon 1999):

C'est pareil pour ma cousine : tout le monde disait que c'est un garçon manqué et après à force d'avoir entendu ça et bien maintenant elle sait que c'est un garçon manqué... la dernière fois, elle m'a dit : "moi, je suis un garçon manqué ». (Dalila)

L'utilisation de cette expression s'inscrit donc dans une relation et un système inégalitaire perçus par les enfants :

Quand ils disent ça [garçon manqué], c'est comme si eux ils étaient supérieurs à elle et qu'elle était pas égale à eux. (Samia).

Pour autant, cette attribution d'identité semble dans les propos des élèves s'adresser à des sujets passifs qu'elle contribuerait à modeler :

Moi, je connaissais une fille, [...] elle a commencé à jouer avec les garçons [...] ; après il y avait des filles qui venaient la voir et lui disaient : " maintenant, tu es plus une vraie fille, tu es un garçon manqué ». Et elle avait tellement marre qu'on lui dise ça, qu'après elle a commencé à mettre des habits de garçons et elle a changé. Après, elle restait que avec des garçons. (Samia)

7. Cette hésitation de Younes est intéressante car elle met en évidence que l'expression garçon manqué, s'inscrit dans un système inégalitaire dans lequel le "masculin " sert de référence, y compris, pour les filles qui s'écartent des normes de ce qui est habituellement considéré comme "féminin ".

8. Dans l'ouvrage, bien que ce ne soit pas explicitement dit, mais juste montré par les images, cet élève a bien compris que l'un des personnages, Auguste, qui aime les poupées et la couture, recherche également des trésors en lisant une carte, avec un T-shirt de pirate. 
La possibilité pour les personnes ainsi désignées de reprendre à leur compte cette identité discriminante pour l'investir de valeurs positives et la revendiquer dans un processus d'émancipation et d'affirmation de soi n'est pas clairement identifiée par les enfants alors même qu'elle semble traverser les anecdotes racontées.

\section{En Cours préparatoire}

La deuxième séance dont nous souhaiterions rendre compte s'est déroulée dans une classe de CP de la même école en janvier 2016. Elle porte sur le livre de T. Lenain illustré par D. Durand, Mademoiselle Zazie a-t-elle un $z i z i$ ? (1996) et fait suite à deux séances au cours desquelles les élèves ont débattu sur les albums T'es fleur ou t'es chou? (Raisson 2008) et Marre du rose (Hense et Green 2009). Dans cette classe, un travail a également été réalisé en début d'année sur l'identification des émotions, notamment les six émotions de base classiquement distinguées en psychologie, soit la joie, la tristesse, la peur, la colère, la surprise, le dégout. La séance est animée par une enseignante de l'école qui n'est pas la titulaire de la classe mais qui a l'habitude de travailler avec ces élèves qu'elle connait bien'.

Mademoiselle Zazie a-t-elle un zizi? raconte l'histoire de Max qui classe les individus en deux catégories hiérarchisées, les «Avec zizi » et les « Sans-zizi ». Il leur assigne, de par ses observations et ses propres pratiques, des gouts et des activités différenciées, mais surtout il les hiérarchise explicitement, les premiers étant plus forts que les secondes, auxquelles il «manque » quelque chose. L'arrivée d'une fille, Zazie, dans sa classe, qui apprécie visiblement les activités et les centres d'intérêts que Max attribue aux garçons et y excelle, amène ce dernier à s'interroger sur l'identité sexuée réelle de cette fille. L'intérêt de ce livre est de questionner les stéréotypes, les préjugés et les inégalités entre les sexes du point de vue d'un enfant qui fait partie du groupe des dominants parce qu'il est un garçon, et se pense clairement comme tel, et de permettre de suivre le cheminement de sa réflexion. En revanche, le livre ne dit rien de ce pense Zazie. À l'inverse de l'héroïne de Marre $d u$ rose, elle semble indifférente aux représentations de son entourage, et notamment de Max, à son sujet.

Lors de cette séance, la professeure opte dès le départ pour un questionnement très différent de celui de l'enseignant précédent : «Alors, qui est-ce qui peut me raconter très rapidement, on ne va pas y passer beaucoup de temps, qui est-ce qui peut me raconter très rapidement l'histoire? ». Elle fait le choix d'orienter les premiers échanges, non sur des enjeux de débat, mais sur la reformulation de l'histoire permettant d'attester à ses yeux de la bonne compréhension des élèves. Ceux-ci produisent quelques éléments en ce sens, jusqu'à ce qu'un élève brule les étapes et s'exclame :

9. Cette séance est également observée par un chercheur qui l'enregistre. 
Max, il raconte n’importe quoi parce que les filles ils ont une zézette et les garçons ils ont un zizi. (Jerry)

Cette affirmation, qui rejoint la conclusion du livre, n'est visiblement pas la réponse espérée par l'enseignante. Bien au contraire, elle souhaite que les élèves passent en revue les différentes étapes du livre et explicitent l'évolution du point de vue de Max :

Toi tu le sais déjà. Mais du coup, pourquoi est-ce que Max [...] voulait savoir si cette fille là elle était pas un peu bizarre, pourquoi est-ce qu'il avait envie de savoir si cette fille là ben par hasard elle aurait pas un zizi ?

Avec cette relance, l'enseignante attend de ses élèves qu'ils-elles lui expliquent ce en quoi Zazie est différente des autres filles. Or, ce n'est pas ce qui se passe, car l'un des garçons de la classe semble croire, à l'instar du héros au début de l'histoire, que Zazie n'est pas réellement une fille et qu'elle aurait effectivement un zizi :

C'est parce que elle a triché [...] quand elle était aux toilettes, elle faisait pipi assis et elle cachait son zizi. (Kamel)

Plutôt que de se saisir de cette opportunité pour commencer le débat, la professeure reste sur des questions de compréhension, persuadée comme elle le dit ensuite dans la séance que " si on comprend pas ça du livre, on peut pas commencer le débat, c'est pas possible ». Pour permettre à Kamel de saisir sa méprise, elle questionne alors la classe de manière de plus en plus insistante et orientée :

Pourquoi est-ce que Max à un moment donné, il a pu penser, comme dit Jerry, il a pu penser quelque chose d'inimaginable, il a pu penser que Zazie ait pu avoir un zizi ? [...] Max il pense que Zazie c'est pas une fille comme les autres. Pourquoi est-ce que Max il pense que Zazie c'est pas une fille comme les autres? Qu'est-ce qui est différent selon lui des autres filles? [...] pourquoi, je repose la question, pourquoi au vu de toutes les activités de Zazie, ce qu'elle aime bien faire, pourquoi est-ce que Max, il s'est mis cette idée qui est complètement fausse dans sa tête, c'est pas vrai, c'est pas possible, d'accord, c'est pas possible que Zazie elle ait un zizi, c'est une fille, mais pourquoi, comment ça se fait que lui il s'est mis ça dans la tête, que c'était possible qu'elle ait un zizi ?

Ce n'est que lorsque la réponse attendue apparait, "parce qu'elle joue pas comme les autres filles ", réponse qu'elle marque d'un "Merci Jerry », qu'elle lance le débat proprement dit.

Elle relit alors le début de l'histoire et demande aux élèves de se positionner par rapport à ce que pense le héros. Ce faisant, elle ne réalise pas que son interrogation porte sur deux niveaux de lecture, la question de l'inégalité de force entre les sexes et celle de son origine :

Est-ce que vous êtes d'accord avec Max ? Est-ce que vous pensez que ça fait depuis très longtemps, depuis le début du monde, ça a toujours été comme 
ça, que les filles, les Sans-zizi, elles sont plus faibles que les garçons ? Est-ce que vous pensez comme Max que ça a toujours été comme ça ? C’est ça qu'il nous raconte quand il nous parle des mammouths, les mammouths c'était il y a très très longtemps. Est-ce que vous pensez comme Max ?

Suite à cette intervention, l'enseignante privilégie dans un premier temps, sans s'en rendre compte, la question de l'origine, pour basculer ensuite abruptement au fil des relances sur celle des inégalités elles-mêmes. Les réponses des élèves, quant à elles, se situent sur l'un ou l'autre niveau ; ce qui donne lieu à un certain nombre de malentendus. Ce type de questionnement, parce qu'il fait référence à des temps anciens qui ne peuvent qu'être imaginés par les enfants, ouvre la porte à la formulation d'opinions qui ne pourront pas être contestées. Pour autant, même sur le temps présent («Est-ce que les garçons sont plus forts que les filles ? »), il favorise l'expression de savoirs de sens commun qui laissent de côté les enjeux proprement littéraires (états mentaux des personnages, interprétation d'une histoire avec retour au texte pour justifier ses positions...) et font du livre le prétexte d'une discussion qui porte sur autre chose que sur l'histoire. Et, de fait, les élèves qui prennent la parole, font part de représentations empreintes de préjugés, que ceux-ci soit conformes à ceux de Max ou en désaccord avec eux, sans que le débat réussisse à s'installer.

L'enseignante s'en aperçoit, semble prendre acte de la complexité de ce qu'elle demande à ses élèves (être capable de déterminer ce qu'ils pensent de ce qu'un personnage pense), et en plein désarroi, décide dans un moment d'hésitation où elle multiplie les interrogations sur des points différents, de décomposer la tâche en revenant sur des questions de compréhension avant de clore la séance :

Qu'est-ce que vous pensez de Max, de son caractère? Non, c'est pas ça que je voulais dire. Qu'est-ce que vous pensez de ce que pense Max des filles? Qu'est-ce qu'il pense des filles Max?

\section{Discussion et conclusion}

Il ne s'agit pas ici d'opposer artificiellement ces deux séances. Elles ont été réalisées dans deux classes et deux niveaux différents, avec des enfants qui n'ont pas le même vécu, la même maturité et ne peuvent pas nécessairement s'engager dans des raisonnements aussi complexes. Elles témoignent également, l'une et l'autre, d'une réflexion amenée à se poursuivre, à être approfondie, et qui ne peut être considérée comme achevée à l'issue d'un temps de débat. Pour autant, l'âge des élèves, en CP et en CM2, pas plus que le choix de l'ouvrage ${ }^{10}$, ne nous semblent être suffisants pour rendre compte de la différence d'engagement des enfants dans

10. L'une des difficultés de Mademoiselle Zazie a-t-elle un zizi ? est en effet de faire porter dans un premier temps la réflexion sur des questions biologiques que les enfants et les adultes peuvent avoir des difficultés à dépasser. 
la discussion et le questionnement. La décision de la deuxième enseignante de conditionner la tenue du débat à la validation de ce qu'elle estime être une bonne compréhension du texte, place en effet les élèves dans un jeu de questions/réponses dans lequel ils n'ont pas la main : seule l'enseignante valide la réponse en dernière instance.

Cette attitude, fréquente dans les séances que nous avons observées, ne laisse pas la possibilité aux enfants de confronter et d'argumenter leurs interprétations à l'aide d'éléments du livre qu'ils auraient l'initiative de mobiliser. Dès lors, ils ne peuvent pas se lancer dans un travail d'interprétation, d'émission d'hypothèses, au risque parfois d'énoncer des contresens sur le texte et de les voir contredits par d'autres camarades, ou d'ouvrir des pistes de réflexion qui se révèleront être des impasses.

L'injonction, dans un second temps, à prendre parti vis-à-vis de ce que pense, Max, le héros, explique également, pour nous, les difficultés rencontrées, notamment en ce qui concerne la possibilité d'envisager le fonctionnement des stéréotypes de sexe de manière dynamique, en lien avec les inégalités qu'ils produisent et la mécanique du récit. L'enseignante semble croire qu'en amenant les élèves à dénoncer les stéréotypes du personnage, elle leur permet de les dénaturaliser. Pourtant, elle ne leur donne pas la possibilité de s'en détacher. De fait, au cours de la séance, ce sont les opinions des élèves qui sont directement convoquées, sans la médiation des personnages du livre.

À l'inverse, l'entrée choisie par l'enseignant de CM2, quoique redondante au cours de la séance, sollicite l'empathie des élèves et le repérage des émotions dans une attitude réflexive vis-à-vis du texte. En partant de la subjectivité des personnages, les enfants réussissent à mettre au jour des rapports de pouvoir dont la perception se complexifie au fur et à mesure des échanges. Il ne s'agit pas ici de donner une réponse unique, de repérer des stéréotypes et de les contrebalancer par des contre-stéréotypes supposés les annuler. Certes, le questionnement mis en œuvre par l'enseignant ne permet pas nécessairement à l'ensemble des élèves de la classe, notamment celles et ceux qui ne parlent pas, d'identifier à chaque fois les points de tension que nous avons relevés. Ce point doit être travaillé. En revanche, il amorce une réflexion sur la manière dont les différences s'articulent aux inégalités, avec comme objectif de stimuler le questionnement et, de fait, la diversité des réponses, des axes d'analyse possibles. Dans cette perspective, la littérature de jeunesse constitue bien plus qu'un outil pour aborder les questions d'égalité des sexes; elle peut être pour les élèves un vecteur pour apporter des réponses à des questions qu'ils et elles se posent sans toujours oser les énoncer, et un moyen de développer chez elles et eux le gout pour la littérature.

Gaël PASQUIER, Cendrine MARRO \& Laurence BRETON 


\section{Références bibliographiques}

- BRETON, L., MARRO, C. \& PASQUIER, G. (2016 à paraitre). Le carnet de littérature, un outil pour réfléchir en classe à l'égalité des sexes ? In $\mathrm{C}$. Mongenot $\&$ S. Ahr (dir.), (D)écrire, prescrire, interdire : les professionnels face à la Littérature de jeunesse aujourd'hui. Paris : ÉMA-AGORA \& CANOPÉ.

- BRUGEILlES, C., CROMER, S. \& PANISSAL, N. (2009). Le sexisme au programme ? Représentation sexuées dans les lectures de référence à l'école. Travail, genre et société, 21(1), 107-129.

- DAFFLON NOVELLE, A. (2006). Littérature enfantine : entre images et sexisme. In A. Dafflon Novelle (dir.), Filles-garçons. Socialisation différenciée ? (pp. 303-324). Grenoble : Presses universitaires de Grenoble.

- ERIBON, D. (1999). Réflexions sur la question gay. Paris : Fayard.

- FERRAND, M. (2004). Féminin Masculin. Paris : La Découverte.

- INSPECTION GÉNÉRALE DE L'ÉDUCATION NATIONALE (IGEN) (2011). L'École maternelle. Paris : Ministère de l'Éducation nationale.

- GOFFMAN, E. (1975). Stigmate. Les usages sociaux des handicaps. Paris : Minuit. - KERGOAT, D. (2005). Rapports sociaux et division du travail entre les sexes. In M. Maruani (dir.). Femmes, genre et société. L'état des savoirs (pp. 94-101). Paris : La Découverte.

- MARRO, C. (2011). De la Mixité à l'égalité. Un cheminement vers l'indépendance à l'égard du genre. Diversité. Ville, École, Intégration, 165, 154-160.

- MARRO, C. (2012). Dépendance-indépendance à l'égard du genre. Penser l'égalité des sexes au-delà de LA différence. Recherche et formation, 69, 65-80.

- PASQUIER, G. (2013). Enseigner l'égalité des sexes à l'école primaire : des pratiques qui se cherchent. In C. Morin-Messabel (dir.). Filles / Garçons. Questions de genre de la formation à l'enseignement (pp. 281-300). Lyon: Presses universitaires de Lyon.

- RICH, A. ([1980] 2010). La Contrainte à l'hétérosexualité et autres essais. Lausanne : Mamamélis \& Nouvelles questions féministes.

\section{Ouvrages de littérature de jeunesse cités}

- HENSE, N. (auteure) \& GREEN, I. (ill.), (2009). Marre du rose. Paris : Albin Michel «Jeunesse».

- LABBÉ, B. \& PUECH, M. (2001). Les Gouters philo. Les garçons et les filles. Paris : Milan « Jeunesse».

- LENAIN, T. (auteur) \& DURAND, D. (ill.) (1996). Madame Zazie a-t-elle un zizi ? Paris : Nathan.

- RAISSON, G. (2008). T'es fleur ou t'es chou? Paris : Rue du Monde. 